\title{
The Chemical Composition of B-type Pulsators: Some Unexpected Results
}

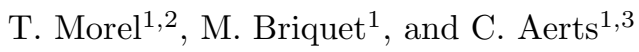 \\ 1 Katholieke Universiteit Leuven, Departement Natuurkunde en Sterrenkunde, \\ Instituut voor Sterrenkunde, Celestijnenlaan 200D, B-3001 Leuven, Belgium \\ 2 European Space Agency (ESA) postdoctoral external fellow \\ 3 Department of Astrophysics, University of Nijmegen, PO Box 9010, 6500 GL \\ Nijmegen, The Netherlands \\ thierry, maryline, conny@ster. kuleuven. be
}

Summary. We present a project aimed at self-consistently deriving the physical parameters and chemical composition of massive pulsators ( $\beta$ Cephei stars, Slowly Pulsating B stars) based on high-resolution optical spectra. Such data will be essential for a proper theoretical interpretation of their oscillation spectrum, but may also contribute in a broader context to our understanding of mixing and diffusion processes in B-type stars. As an illustration, our first results reveal the existence of core-processed material at the surface of some slowly-rotating $\beta$ Cephei stars which is not predicted by current evolutionary models including rotation.

\section{B-type Pulsators and the Rationale of the Project}

The $\beta$ Cephei and slowly pulsating B stars (SPBs) are two well-established classes of pulsating variables found in the upper part of the main sequence. The $\beta$ Cephei stars (B0-B3) pulsate through low radial order $p / g$ modes with periods in the range 2-12 hours, while SPBs (B2-B9) exhibit high radial order $g$ modes and longer periods in the range $0.5-5$ days.

Dramatic advances in our knowledge of the pulsational properties of these objects are presently being made from multisite, ground-based campaigns or from space (e.g. MOST). In turn, theoretical modelling of these data is expected to give unique insights into some fundamental, yet poorly-known parameters of B-type stars, such as the amount of convective core overshooting or the rotation profile in the interior (e.g. [1]). Accurate estimates of the physical parameters and metallicity of these targets are needed to constrain the theoretical models and, ultimately, for correct inferences regarding their internal structure.

This has motivated us to launch an NLTE abundance study of a large sample of prime B-type targets for asteroseismology using fully line-blanketed Kurucz atmospheric models, the line formation codes DETAIL/SURFACE and high-resolution optical spectra obtained with a variety of echelle spectrographs offering a resolving power $R \sim 50000$ (mainly CORALIE, ELODIE and FEROS). Our analysis is based on a large number of time-resolved spectra covering in most cases the entire oscillation cycle of the stars. 


\section{Nitrogen Excess in Slowly-rotating $\beta$ Cephei Stars: Deep Mixing or Diffusion?}

Our initial study of a number of prototypical $\beta$ Cephei stars [6] reveals that their photospheric metal content is indistinguishable from the values previously reported for early B dwarfs in the solar neighbourhood. However, we clearly detect in some objects an unexpected nitrogen excess accompanied by a strong boron depletion, as expected if core-processed material were dredged up to the surface by rotationally-induced mixing. Although appealing, one serious difficulty with this interpretation lies in the fact that the equatorial velocities of most $\mathrm{N}$-enriched stars appear well below what is required by the most recent evolutionary models including rotation (e.g. [4]). Alternatively, recent theoretical work suggests that diffusion effects might be invoked [2]. In any case, any attempt to explain this phenomenon should account for the fact that a magnetic field of up to a few hundreds Gauss has been detected in most N-rich stars (see, e.g. [5]).

\section{Future Developments}

We are currently carrying out an abundance study of about $3 / 4$ of all confirmed SPBs ( 20 stars) using the same techniques (Briquet et al., in prep.). Most stars in this sample have already been the subject of sensitive magnetic field measurements [5], a fact which will allow us to further investigate the incidence of chemical peculiarities as a function of the field strength. Preliminary results [3] also suggest the existence of N-rich, B-depleted stars in this class of objects, e.g. the magnetic star $\zeta$ Cas with a polar field strength $B_{p}=340 \pm 90 \mathrm{G}$ and a rotational velocity of only about $55 \mathrm{~km} \mathrm{~s}^{-1}$ [8].

A vast number of B stars will be observed during the course of the asteroseismology programme of the CoRoT mission. High-resolution optical spectra have so far been obtained for $\sim 200$ stars in the zones of the sky which will be surveyed by the satellite as part of the ground-based preparatory campaign. We plan to analyze the full sample using (semi-)automated, spectral synthesis techniques (see [7] for the very first results).

\section{References}

1. C. Aerts, A. Thoul, J. Daszyńska, et al.: Science 300, 1926 (2003)

2. P.-O. Bourge, S. Théado, A. Thoul: MNRAS, submitted (2006)

3. M. Briquet, T. Morel: CoAst, 148, in press (2007)

4. A. Heger, N. Langer: ApJ, 544, 1016 (2000)

5. S. Hubrig, M. Briquet, M. Schöller, et al.: MNRAS 369, L61 (2006)

6. T. Morel, K. Butler, C. Aerts, et al.: A\&A 457, 651 (2006)

7. T. Morel, C. Aerts: CoAst, 148, in press (2007)

8. C. Neiner, V. C. Geers, H. F. Henrichs, et al.: A\&A 406, 1019 (2003) 